\title{
RELATIONS INVOLVING THE FORWARD SCATTERING AMPLITUDE IN A FERMI GAS
}

\author{
by B. A. JACOBSOHN *) \\ Instituut voor theoretische fysica der Rijksuniversiteit, Utrecht, Nederland, and \\ CERN, Geneva, Switzerland
}

\section{Synopsis}

Several exact relationships are given for the ground state of a Fermi gas with interaction. These involve the two-particle forward scattering amplitude and the effective mass at the Fermi surface.

Hugenholtz and Van Hove ${ }^{1}$ ) have demonstrated that a relationship exists between the energy of a particle or hole at the top of the Fermi sea and the derivative with respect to the density of the energy per unit volume, for the ground state of a Fermi gas with interaction. Their theorem is exact in the sense of being true for each finite order in the interaction. Their method involves an examination in terms of diagrams of the consequences of two alternative modes of viewing a system of $(N \pm 1)$ particles: on the one hand as a "normal" Fermi sea of $N$ particles plus an extra particle (or hole); on the other hand as a "new" sea of $(N \pm 1)$ particles. As they show, this procedure can be expressed in terms of cutting an internal line of each disconnected ground state diagram.

Further results can be found by the analogous examination of systems with $(N \pm 2)$ particles. This is equivalent to the cutting of each ground state diagram at two internal lines, or each single particle diagram once. An example of such successive cutting is shown in Figure 1. One would suspect that a relationship involving the forward scattering amplitude for two particles can be found in this way. This turns out to be the case.

The fundamental theorem which has been shown to hold to all orders can be written

$$
\begin{aligned}
& \bar{E}_{k}\left(k^{\prime}\right\rangle-\bar{E}_{k}(0)=2 \Omega^{-1}\left\langle k k^{\prime}\left|T_{p p}\right| k k^{\prime}\right\rangle \\
& -\bar{E}_{k}(m)+\bar{E}_{k}(0)=2 \Omega^{-1}\left\langle k m\left|T_{p h}\right| k m\right\rangle=\bar{E}_{m}(k)-\bar{E}_{m}(0), \\
& -\bar{E}_{m}\left(m^{\prime}\right)+\bar{E}_{m}(0)=2 \Omega^{-1}\left\langle m m^{\prime}\left|T_{h h}\right| m m^{\prime}\right\rangle .
\end{aligned}
$$

In the above $|\boldsymbol{k}|,\left|\boldsymbol{k}^{\prime}\right|>p_{\boldsymbol{F}}>|\boldsymbol{m}|,\left|\boldsymbol{m}^{\prime}\right|, \bar{E}_{k}(0)$ is the energy (in general complex) of a particle in state $\boldsymbol{k}$ in the presence of the original Fermi sea;

*) On leave from the University of Washington, Seattle, U.S.A. 
$\bar{E}_{k}\left(k^{\prime}\right)$ is the same quantity when the sea contains an additional particle in state $\boldsymbol{k}^{\prime}$; and $\overline{\boldsymbol{E}}_{k}(\boldsymbol{m})$ is the same when the sea has a hole in the state $\boldsymbol{m}$. $\Omega$ is the volume. The right-hand sides of (1.1), (1.2) and (1.3) contain respectively the forward particle-particle, particle-hole, and hole-hole scattering amplitudes in the presence of the Fermi sea.

This theorem has several useful applications. Some of them involve differentiation with respect to the density, which in turn means comparing

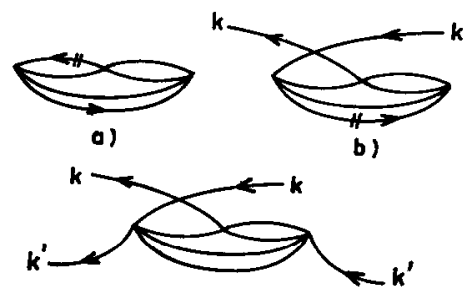

e)

Fig. 1. a is a typical ground state diagram in which an internal particle line has been cut to give b, a single particle diagram. A second cut can give a two-particle forward scattering diagram, as in c.

two Fermi spheres of slightly different sizes as in reference1). One finds the following exact results:

a) In the limit of low density,

$\left(\frac{\mathrm{d} \bar{E}_{k}}{\mathrm{~d} \rho}\right)_{\rho=0}=$

$=2\left\langle k 0\left|T_{\text {vac. }}\right| k 0\right\rangle_{\text {av. }}=-\frac{4 \pi}{M k}\left\{\left(\Sigma_{L \text { even }}+3 \Sigma_{L \text { odd }}\right)(2 L+1) \mathrm{e}^{i \delta L} \sin \delta_{L}\right\}$,

in which $\delta_{L}$ is a phase shift in vacuum for two particles with relative momentum $k / 2$. This has been written out explicitly for spin-independent forces, and illustrates the normalization of $\boldsymbol{T}$.

b) Relations among the scattering amplitudes for particles and holes follow from comparisons of the lefthand sides of equations (1.1)-(1.3):

$$
\begin{aligned}
\left\langle k p_{F}\left|T_{p p}\right| k p_{F}\right\rangle & =\left\langle k p_{F}\left|T_{p h}\right| k p_{F}\right\rangle ; \\
\left\langle m p_{F}\left|T_{h \boldsymbol{h}}\right| m p_{F}\right\rangle & =\left\langle m p_{F}\left|T_{h p}\right| m p_{F}\right\rangle .
\end{aligned}
$$

If both momenta are at the Fermi surface, then

$$
T_{p p}=T_{h h}=T_{p h} .
$$

These results are not completely trivial because of the complications introduced by the self-energy insertions in the external lines.

c) A combination of our result with the Hugenholtz-Van Hove theorem yields

$$
\frac{\mathrm{d}^{2}}{\mathrm{~d} \rho^{2}}\left(\frac{E_{0}}{\Omega}\right)=\frac{\pi^{2}}{M_{F^{*}} p_{F}}+2\left\langle\boldsymbol{p}_{F} p_{F}{ }^{\prime}|T| p_{F} p_{F^{\prime}}\right\rangle_{\mathrm{av}}
$$


in which $E_{0}$ is the ground state energy and $M_{F}{ }^{*}$ is the effective mass of a particle at the Fermi surface. The spins and the directions of $\boldsymbol{p}$ and $\boldsymbol{p}^{\prime}$ are to be averaged.

d) In accordance with (4), we have omitted subscripts $\boldsymbol{p}$ or $\boldsymbol{h}$ on $\boldsymbol{T}$ in (5), and have therefore anticipated the result

$$
\left(M_{F}^{*}\right)_{\text {particle }}=\left(M_{F}^{*}\right)_{\text {hole }}
$$

e) Combining the Galilean invariance of the system with equation (1.2), an independent relation involving a different average over $T$ is found:

$$
\frac{\pi^{2}}{p_{F}}\left(\frac{1}{M}-\frac{1}{M_{F}{ }^{*}}\right)=2\left\{\left\langle\boldsymbol{p}_{F} \boldsymbol{p}_{F^{\prime}}|T| \boldsymbol{p}_{F} \boldsymbol{p}_{F^{\prime}}\right\rangle \cos \theta\right\}_{\mathrm{av}}
$$

The angle between $\boldsymbol{p}$ and $\boldsymbol{p}^{\prime}$ is denoted by $\theta$.

The scattering amplitude cannot be eliminated from equations (5) and (7) because of the different averages which appear.

Certain of these relations occur in Landau's semiphenomenological theory of a Fermi liquid ${ }^{2}$ ). The present work constitutes a proof of these relations within the framework of microscopic many-body theory.

A detailed paper on this subject is being prepared for submission to Physica.

REFERENCES

1) Hugenholtz, N. M. and Van Hove, L., Physica 24 (1958) 363.

2) Landau, L. D., Soviet Physics JETP 3 (1957) 920. 